\title{
Comparison of routine hematological indicators of liver and kidney function, blood count and lipid profile in healthy people and stroke patients
}

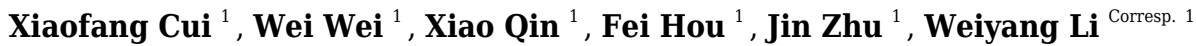 \\ 1 Jining Medical University, Jining, China \\ Corresponding Author: Weiyang Li \\ Email address: 163.Iwy@163.com
}

Background and methods: Stroke has become a major public health problem worldwide. In this paper, we carried out statistical analysis, correlation analysis and principal component analysis to evaluate the clinical value of routine hematological indicators in early diagnosis of ischemic stroke using R language. Results: For the full blood count comparisons, stroke patients had obvious differences in the distribution width of red blood cells (RDW-CV), average distribution width of red blood cells (RDW-SD), mean hemoglobin concentrations, platelet large cell ratio, mean platelet volume and lymphocytes. Patients with ischemic stroke also exhibited different degrees of abnormalities in liver function test. With respect to renal function, stroke patients had obvious changes in uric acid and urea levels. Finally, when comparing the lipid profile, triglyceride concentrations were increased and high-density lipoprotein cholesterol concentrations were reduced in stroke patients. In addition, correlation analysis among these clinical indicators indicated that there were both common characteristics and differences between patients and health controls. Furthermore, the results of principal component analysis indicated that these clinical indicators could distinguish patients from the healthy controls. Conclusion: Conventional hematological clinical indicators, such as liver function, renal function, full blood count and lipid concentration profiles highly correlated with the occurrence of ischemic stroke. Therefore, the detection and analyzation of these clinical indicators are of great significance for the prediction of ischemic stroke. 
1 Comparison of routine hematological indicators of liver and kidney function, blood count and lipid profile in healthy people and stroke patients

3

4 Xiaofang Cui ${ }^{1,2}$, Wei Wei ${ }^{1,3}$, Xiao Qin ${ }^{1,3}$, Fei Hou ${ }^{1,3}$ Jin Zhu $^{1,2}$,Weiyang Li $^{1,3 *}$

51 Jining Medical University, Jining, Shandong 272067, China

62 Shandong Key Laboratory of Behavioral Medicine, School of Mental Health, Jining Medical

7 University, Jining, Shandong 272067, China

83 Collaborative Innovation Center for Birth Defect Research and Transformation of Shandong

9 Province, Jining Medical University, Jining, Shandong 272067, China

10

11 * Correspondence should be addressed to Weiyang Li, 163.1wy@163.com.

12 


\section{Abstract}

Background and methods: Stroke has become a major public health problem worldwide. In this paper, we carried out statistical analysis, correlation analysis and principal component analysis to evaluate the clinical value of routine hematological indicators in early diagnosis of ischemic stroke using R language. Results: For the full blood count comparisons, stroke patients had obvious differences in the distribution width of red blood cells (RDW-CV), average distribution width of red blood cells (RDW-SD), mean hemoglobin concentrations, platelet large cell ratio, mean platelet volume and lymphocytes. Patients with ischemic stroke also exhibited different degrees of abnormalities in liver function test. With respect to renal function, stroke patients had obvious changes in uric acid and urea levels. Finally, when comparing the lipid profile, triglyceride concentrations were increased and high-density lipoprotein cholesterol concentrations were reduced in stroke patients. In addition, correlation analysis among these clinical indicators indicated that there were both common characteristics and differences between patients and health controls . Furthermore, the results of principal component analysis indicated that these clinical indicators could distinguish patients from the healthy controls. Conclusion: Conventional hematological clinical indicators, such as liver function, renal function, full blood count and lipid concentration profiles highly correlated with the occurrence of ischemic stroke. Therefore, the detection and analyzation of these clinical indicators are of great significance for the prediction of ischemic stroke. 


\section{Introduction}

Stroke is an acute cerebrovascular disease characterized by focal neurological deficits, which is due to ischemia in 60-80\% of cases (Doberstein et al. 2017; Global Burden of Disease Study 2015). Stroke is common, with an incidence that increases yearly, and carries a high disability and mortality rate. Nowadays, stroke has become a major public health problem all over the world (Doberstein et al. 2017; Feigin et al. 2015; Global Burden of Disease Study 2015). Thus, increasing our understanding of the clinical indicators for this disease is essential to improve the ability to predict stroke risk, as well as to reduce its incidence and associated disabilities. It is known that independent risk factors for stroke include age, sex, body mass index, positive family history, cigarette smoking, alcohol consumption, hyperlipidemia, hypertension, diabetes and heart disease (Braillon 2018; Guzik \& Bushnell 2017; Kim \& Kim 2018; Lan et al. 2018). These factors may all cause changes in physiological functions thereby leading to abnormalities in renal or hepatic function, or blood count or lipid profile. Recent advances have been made in identifying biomarkers of acute ischemic stroke (Arsava et al. 2011; Chatzikonstantinou et al. 2013; Maier et al. 2013), but only a few systematic studies have been undertaken on the relationship between test indicators, such as liver function, renal function or full blood count and acute ischemic stroke (Turcato et al. 2017a; Turcato et al. 2017b). These bloods tests are routinely performed on hospital admission, and are minimally invasive and of low cost. In this study, we systematically evaluated the results of routine clinical blood tests to determine if there were obvious differences between patients with acute ischemic stroke and healthy individuals. Identification of easily performed clinical indicators that predict stroke is of great significance for early diagnosis and management of stroke.

\section{Materials and methods}

Forty-one patients with acute ischemic stroke who were admitted to the Department of Neurology at Jining People's Hospital from April to December 2018 were enrolled as the disease group, and 80 healthy subjects were included as controls. The study had been approved by the Ethics Review Committee of the Jining Medical University and written informed consent was 
obtained from all subjects. For the stroke group, the sole inclusion criterion was that the patient was diagnosed with a first ischemic stroke event; patients with stroke accompanied by severe complications were excluded. Healthy individuals without obvious diseases were included in the healthy control group. Patients and healthy individuals with incomplete clinical data were excluded. Next, we assessed 1) renal function, 2) liver function, 3) full blood count, and 4) lipid concentrations. Data were processed by R language software, and the differences between two groups were examined by Student's $\mathrm{t}$-test. Correlation analyse were carried out according to the method of Pearson correlation coefficient. Principal component analysis (PCA) was performed based on the $\mathrm{R}$ language software.

\section{Results}

\subsection{Comparison of full blood count}

Obvious differences were observed between the two groups in the distribution width of red blood cells (RDW-CV), average distribution width of red blood cells (RDW-SD), average hemoglobin concentration, average platelet volume, large platelet ratio and lymphocyte count as shown in Figure 1. Compared with healthy individuals, the distribution of red blood cells in stroke patients was elevated, with increased mean hemoglobin concentration. Furthermore, compared with healthy individuals, the mean platelet volume in stroke patients was slightly lower $(\mathrm{P}<0.01)$, while the large platelet ratio was elevated $(\mathrm{P}<0.01)$. The lymphocyte concentration in stroke patients was lower compared to that in healthy individuals $(\mathrm{P}<0.01)$.

3.2 Comparison of liver function, lipid concentrations and renal function

We compared the liver function, renal function and lipid profile test results on admission between the two groups and the results are shown in Figures 2 and 3. First, the liver function test results demonstrated that the albumin, albumin /globulin and total protein concentrations in stroke patients was lower than in healthy individuals $(\mathrm{P}<0.01)$, while the globulin concentration was higher $(\mathrm{P}>0.05)$. Further, stroke patients had lower indirect (unconjugated) and total bilirubin compared with the healthy group $(\mathrm{P}<0.01)$. Second, comparison of the differences in blood lipid 
106

107

108

109

110

111

112

113

114

115

116

117

118

119

120

121

122

123

124

concentrations between the two groups revealed that the triglyceride concentration was higher and the high-density lipoprotein cholesterol concentration lower in stroke patients $(\mathrm{P}<0.01)$. The differences in concentrations of the other lipids were not significant between the two groups $(\mathrm{P}>$ 0.05). Third, for evaluation of renal function, the uric acid concentration was notably reduced in the disease group, while the urea concentration was increased $(\mathrm{P}<0.01)$.

3.3 Correlation and principal component analyses of the clinical indicators between the two groups Since multiple clinical indicators are associated with morbidity and mortality in patients with ischemic stroke, we correlated the various clinical indicators as shown in Figure 4. Our findings indicate that there were correlations between the indicators in the disease group and in the healthy group, and that the correlation between the two groups was basically consistent with each other. However, the correlation of some indicators exhibited remarkable differences between the two groups. For instance, the albumin/globulin ratio was negatively correlated with total protein in healthy individual, however this was not significant in the stroke group. Further, the high-density lipoprotein cholesterol concentration in the healthy group was negatively correlated with triglyceride concentration, however this was not observed in the patient group. In addition, there was an obvious negative association between mean cell hemoglobin and RDW-CV in the healthy group, which was not found in the patient group. In the healthy group, there was a positive correlation between mean cell hemoglobin concentration and mean cell hemoglobin, while in the patient group they were not significantly associated (Figures $4 a, 4 b, 4 c$ ). Due to the large number of clinical indicators and the significant differences in multiple indicators between the healthy group and the stroke group, a comprehensive principal component analysis was performed on the liver function tests, renal function tests, full blood count and lipid concentration profile. This clearly distinguished the healthy group from the disease group through differential indicators as shown in Figure 5. 
133

134

135

136

137

138

139

140

141

142

143

144

145

146

147

148

149

150

151

152

153

154

155

156

157

158

159

\section{Discussion}

Routine hematological testing in the clinical setting, that is important for diagnosis and management of diseases, includes evaluation of liver function, renal function, full blood count and lipid concentration profile. Although no systematic studies on the correlation between these routine clinical indicators and ischemic stroke have been performed either in China or abroad, a few reports have been published that evaluated the relationship between routine blood tests and stroke. Recent reports have been published on the relationship between the neutrophil/lymphocyte ratio (Xue et al. 2017; Zhang et al. 2011), red blood cell distribution width and stroke prognosis at home and abroad (Kim et al. 2012a; Turcato et al. 2017b). In 2017, Turcato et al.(Turcato et al. 2017a) found that determination of red blood cell distribution width could provide guidance on the risk of cardiovascular and cerebrovascular diseases. Cao et al. (Cao et al. 2011) and Zhang et al.(Zhang et al. 2012) discovered that both the bilirubin concentration and hypertension were independently and negatively correlated with other risk factors. Since hypertension is one of the main risk factors for stroke, the occurrence of stroke may correlate with the abnormalities in liver function. Given that renal dysfunction often occurs during stroke episodes, a correlation between abnormal renal function and stroke may be possible. Zhang et al. (Zhang et al. 2013; Zhang et al. 2012) confirmed a strong correlation between elevated urea concentrations and poor prognosis in stroke patients. Moreover, many studies have reported a relationship between blood lipid concentrations and stroke (Cui et al. 2012; Lewis \& Segal 2010). Dyslipidemia is accepted as one of the risk factors for stroke because it can cause atherosclerosis, which is one of the pathological processes underlying stroke(Kim et al. 2012b). Therefore, the blood lipid concentration profile of stroke patients is usually abnormal.

In order to determine the differences in the above-mentioned clinical indicators between stroke patients and the healthy controls, we completed a separate analysis on more than forty indicators that were evaluated in patients with ischemic stroke. Red blood cell width distribution, mean erythrocyte hemoglobin content, platelet count, mean platelet volume, platelet large cell ratio and lymphocyte concentration were among those parameters that were notably different in stroke 
160

161

162

163

164

165

166

167

168

169

170

171

172

173

174

175

176

177

178

179

180

181

182

183

184

185

186

187

188

patients when compared with healthy controls. Liver function test results demonstrated abnormalities in liver function protein and bilirubin metabolism in stroke patients compared with healthy controls. We also observed that the serum uric acid concentration was decreased in stroke patients, while the urea concentration was remarkably elevated. This was consistent with previous findings (Zhang et al. 2013). In addition, the triglyceride and high-density lipoprotein cholesterol concentrations of stroke patients were significantly different from those of healthy controls. Therefore, measurement of these indicators may help predict or identify stroke. Through correlation studies, we explored the correlations between the indicators in both the stroke group and the healthy group, and significant differences were observed. The differences in correlations may be of great significance for predicting stroke. Subsequently, principal component analysis was conducted on these indicators, and obvious differences were identified between the two groups. We found that these indicators could distinguish the stroke patients from the healthy controls. This comprehensive analysis of these indicators showed significant differences between the two groups, thereby presenting the ability to distinguish the two groups, and provide a theoretical basis for using these conventional indicators to diagnose stroke. In summary, early detection of changes in routine hematological indicators of liver and renal function, along with blood count and lipid concentrations in patients with ischemic stroke may have significant potential for early diagnosis, dynamic monitoring and evaluation of therapeutic effects.

\section{Acknowledgements}

This study was supported by Research from the Natural Science Foundation of Shandong Province (ZR2018PH018) and the Jining Medical University (JY2017JS004).

\section{Ethical approval and informed consent}

All procedures performed in this study involving human participants were in accordance with the ethical standards of the institutional research committee and with the 1964 Helsinki Declaration and its later amendments or comparable ethical

standards. The study was approved by the Ethics Review Committee of the Jining Medical University and informed consent was obtained from all subjects.

\section{Author Contributions}


Xiaofang Cui and Weiyang Li conceived and designed the research. W.W, X.Q, F.H and J.Z performed the experiments and interpreted the data. X.F.C., W.Y.L wrote the main manuscript. All authors reviewed the manuscript.

Competing Interests: The authors declare no competing interests.

\section{References}

Arsava EM, Furie KL, Schwamm LH, Sorensen AG, and Ay H. 2011. Prediction of early stroke risk in transient symptoms with infarction: relevance to the new tissue-based definition. Stroke 42:2186-2190. 10.1161/STROKEAHA.110.604280

Braillon A. 2018. Smoking is a cause of ischaemic stroke, not a risk factor! Eur J Neurol 25:e49. 10.1111/ene.13585 Cao J, Sodhi K, Inoue K, Quilley J, Rezzani R, Rodella L, Vanella L, Germinario L, Stec DE, Abraham NG, and Kappas A. 2011. Lentiviral-human heme oxygenase targeting endothelium improved vascular function in angiotensin II animal model of hypertension. Hum Gene Ther 22:271-282. 10.1089/hum.2010.059

Chatzikonstantinou A, Wolf ME, Schaefer A, and Hennerici MG. 2013. Risk prediction of subsequent early stroke in patients with transient ischemic attacks. Cerebrovasc Dis 36:106-109. 10.1159/000352060

Cui R, Iso H, Yamagishi K, Saito I, Kokubo Y, Inoue M, Tsugane S, and Group JS. 2012. High serum total cholesterol levels is a risk factor of ischemic stroke for general Japanese population: the JPHC study. Atherosclerosis 221:565-569. 10.1016/j.atherosclerosis.2012.01.013

Doberstein CA, Torabi R, Yan SC, McTaggart R, Doberstein C, and Jayaraman M. 2017. Current Strategies in the Surgical Management of Ischemic Stroke. R I Med J (2013) 100:25-29.

Feigin VL, Krishnamurthi RV, Parmar P, Norrving B, Mensah GA, Bennett DA, Barker-Collo S, Moran AE, Sacco RL, Truelsen T, Davis S, Pandian JD, Naghavi M, Forouzanfar MH, Nguyen G, Johnson CO, Vos T, Meretoja A, Murray CJ, Roth GA, Group GBDW, and Group GBDSPE. 2015. Update on the Global Burden of Ischemic and Hemorrhagic Stroke in 1990-2013: The GBD 2013 Study. Neuroepidemiology 45:161-176. 10.1159/000441085

Global Burden of Disease Study C. 2015. Global, regional, and national incidence, prevalence, and years lived with disability for 301 acute and chronic diseases and injuries in 188 countries, 1990-2013: a systematic analysis for the Global Burden of Disease Study 2013. Lancet 386:743-800. 10.1016/S01406736(15)60692-4

Guzik A, and Bushnell C. 2017. Stroke Epidemiology and Risk Factor Management. Continuum (Minneap Minn) 23:15-39. 10.1212/CON.0000000000000416

Kim J, Kim YD, Song TJ, Park JH, Lee HS, Nam CM, Nam HS, and Heo JH. 2012a. Red blood cell distribution width is associated with poor clinical outcome in acute cerebral infarction. Thromb Haemost 108:349-356. 10.1160/TH12-03-0165

Kim SJ, Park YG, Kim JH, Han YK, Cho HK, and Bang OY. 2012b. Plasma fasting and nonfasting triglycerides and highdensity lipoprotein cholesterol in atherosclerotic stroke: different profiles according to low-density lipoprotein cholesterol. Atherosclerosis 223:463-467. 10.1016/j.atherosclerosis.2012.05.011

Kim W, and Kim EJ. 2018. Heart Failure as a Risk Factor for Stroke. J Stroke 20:33-45. 10.5853/jos.2017.02810 Lan DH, Jiang C, Du X, He L, Guo XY, Zuo S, Xia SJ, Chang SS, Wen SN, Wu JH, Ruan YF, Long DY, Tang RB, Yu RH, 
Sang CH, Bai R, Liu N, Jiang CX, Li SN, Dong JZ, Lip GYH, Chen AH, and Ma CS. 2018. Female Sex as a Risk Factor for Ischemic Stroke and Systemic Embolism in Chinese Patients With Atrial Fibrillation: A Report From the China-AF Study. J Am Heart Assoc 7:e009391. 10.1161/JAHA.118.009391

Lewis A, and Segal A. 2010. Hyperlipidemia and primary prevention of stroke: does risk factor identification and reduction really work? Curr Atheroscler Rep 12:225-229. 10.1007/s11883-010-0117-4

Maier IL, Bauerle M, Kermer P, Helms HJ, and Buettner T. 2013. Risk prediction of very early recurrence, death and progression after acute ischaemic stroke. Eur J Neurol 20:599-604. 10.1111/ene.12037

Turcato G, Cappellari M, Follador L, Dilda A, Bonora A, Zannoni M, Bovo C, Ricci G, Bovi P, and Lippi G. 2017a. Red Blood Cell Distribution Width Is an Independent Predictor of Outcome in Patients Undergoing Thrombolysis for Ischemic Stroke. Semin Thromb Hemost 43:30-35. 10.1055/s-0036-1592165

Turcato G, Cervellin G, Cappellari M, Bonora A, Zannoni M, Bovi P, Ricci G, and Lippi G. 2017b. Early function decline after ischemic stroke can be predicted by a nomogram based on age, use of thrombolysis, RDW and NIHSS score at admission. J Thromb Thrombolysis 43:394-400. 10.1007/s11239-016-1456-y

Xue J, Huang W, Chen X, Li Q, Cai Z, Yu T, and Shao B. 2017. Neutrophil-to-Lymphocyte Ratio Is a Prognostic Marker in Acute Ischemic Stroke. J Stroke Cerebrovasc Dis 26:650-657. 10.1016/j.jstrokecerebrovasdis.2016.11.010

Zhang J, Peng Y, Fan H, Chen M, Xu T, and Zhang Y. 2011. Blood pressure and early clinical outcome among acute ischemic stroke patients. Can J Neurol Sci 38:225-229.

Zhang Y, Churilov L, Meretoja A, Teo S, Davis SM, and Yan B. 2013. Elevated urea level is associated with poor clinical outcome and increased mortality post intravenous tissue plasminogen activator in stroke patients. J Neurol Sci 332:110-115. 10.1016/j.jns.2013.06.030

Zhang ZY, Bian LQ, Kim SJ, Zhou CC, and Choi YH. 2012. Inverse relation of total serum bilirubin to coronary artery calcification score detected by multidetector computed tomography in males. Clin Cardiol 35:301-306. 10.1002/clc.21964

Figure Legends

Fig 1. Comparison of routine blood indicators

Fig1 legend: Red blood cell distribution width (RDW-CV); Mean Red blood Cell distribution 
270

271

272

273

274

275

276

277

278

279

280

281

282

283

284

285

286

287

288

289

290

291

292

293

294

295

296

297

298

299

300

301

width (RDW-SD); Mean corpuscular hemoglobin (MCH); Mean corpuscular hemoglobin concentration (MCHC); Platelet (PLT); Mean platelet volume (MPV); Platelet large cell ratio (PLCR); Lymphocyte (LY).Green represents the patient group; Tangerine represents the normal group in the boxplot. The Student's t-test was used to calculate the P value between groups.

Fig 2 Comparison of liver function and blood lipid indicators

Fig2 legend: Albumin (ALB); Globulin (GLB); Albumin/Globulin (A/G); Total protein (TP), Indirect bilirubin (IBIL); Direct bilirubin (DBIL); Total bilirubin (TBIL); Triglyceride (TG);High density lipoprotein cholesterol (HDL-C). Green represents the patient group; Tangerine represents the normal group in the boxplot. The Student's t-test was used to calculate the $\mathrm{P}$ value between groups.

Fig 3 Comparison of renal function

Fig legend: Uric acid (UA);Blood urea nitrogen (BUN). Green represents the patient group; Tangerine represents the normal group in the boxplot. The Student's t-test was used to calculate the $\mathrm{P}$ value between groups.

Fig 4 Correlation of clinical indicators

Fig legend: (a)Normal represents the correlation of clinical indicators in health group; the color represents the association strength. The Pearson Correlation Coefficient was used to calculate the correlation. (b)Patient represents the correlation of clinical indicators in stroke patients; the color represents the association strength. The Pearson Correlation Coefficient was used to calculate the correlation. (c) Difference represents the difference value of the correlation coefficient between Normal and Patient. The color represents difference value.

Fig 5 Principal component analysis plot

\section{Supplementary Material}

Table S1 Health group information

TableS2 Case group information 


\section{Figure 1}

Comparison of routine blood indicators

Red blood cell distribution width (RDW-CV); Mean Red blood Cell distribution width (RDW-SD); Mean corpuscular hemoglobin (MCH); Mean corpuscular hemoglobin concentration (MCHC); Platelet (PLT); Mean platelet volume (MPV); Platelet large cell ratio (P-LCR); Lymphocyte (LY).Green represents the patient group; Tangerine represents the normal group in the boxplot. The Student's t-test was used to calculate the $\mathrm{P}$ value between groups.
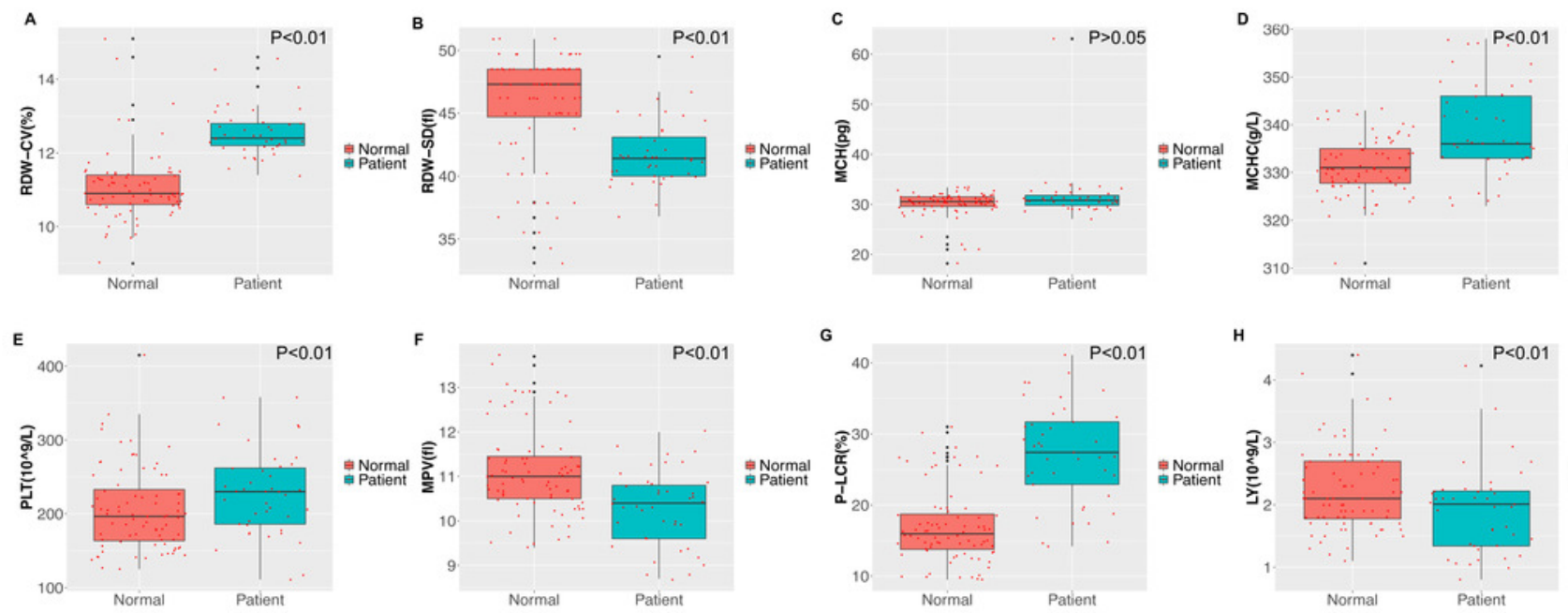

i Normal
i Patient 


\section{Figure 2}

Comparison of liver function and blood lipid indicators

Albumin (ALB); Globulin (GLB); Albumin/Globulin (A/G); Total protein (TP), Indirect bilirubin (IBIL); Direct bilirubin (DBIL); Total bilirubin (TBIL); Triglyceride (TG); High density lipoprotein cholesterol (HDL-C). Green represents the patient group; Tangerine represents the normal group in the boxplot. The Student's t-test was used to calculate the P value between groups.

A

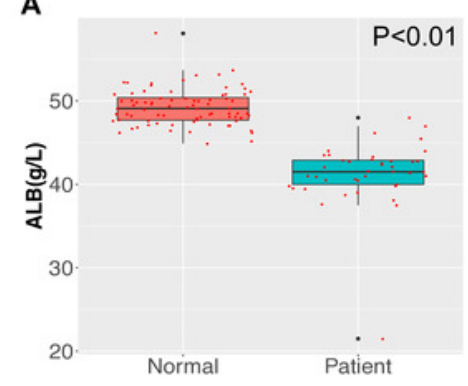

D
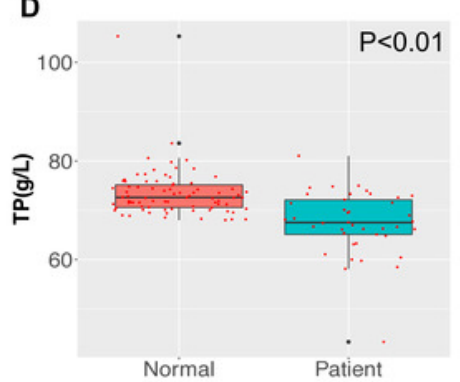

G

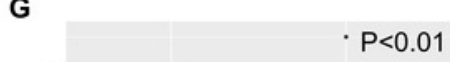

40

ङ

10
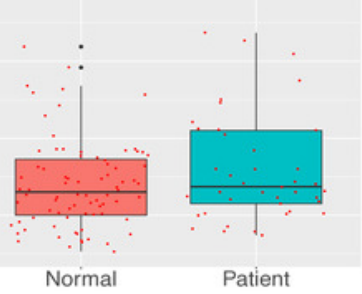

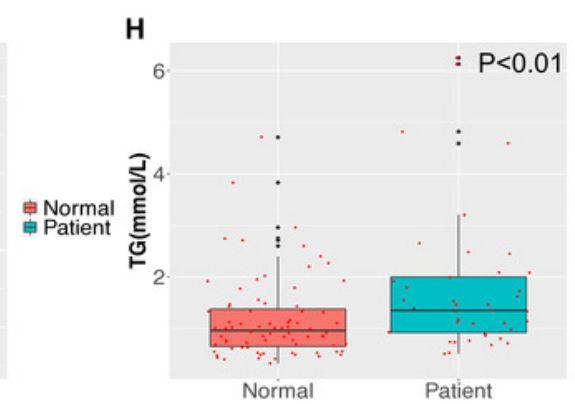

E
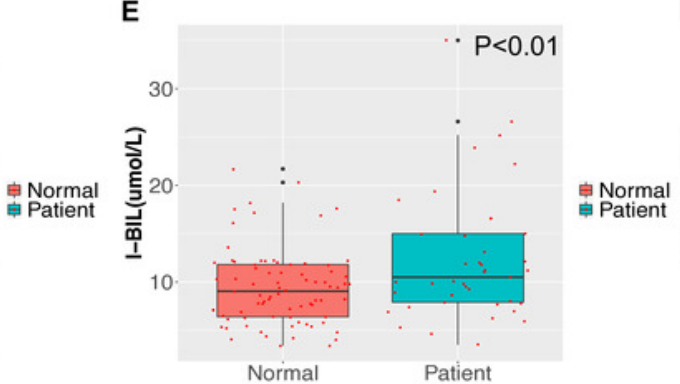

F

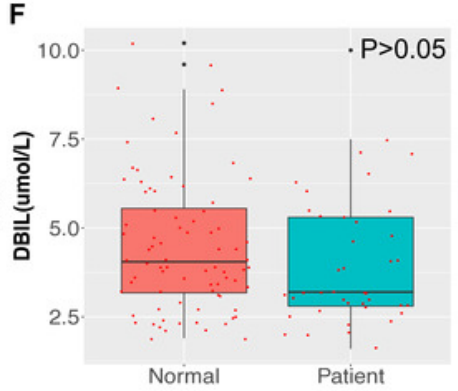

帛 Normal Patient

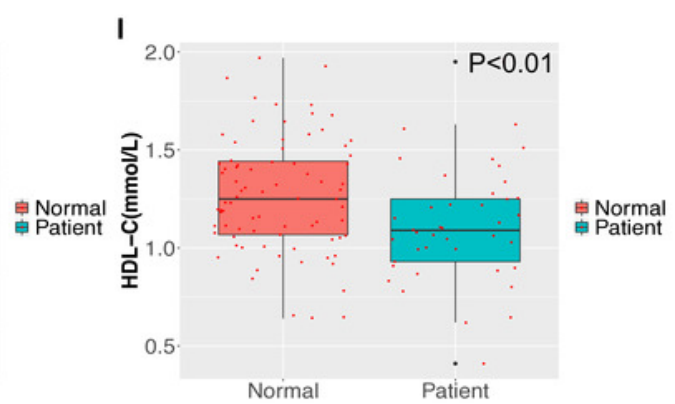


Figure 3

Comparison of renal function

Uric acid (UA);Blood urea nitrogen (BUN). Green represents the patient group; Tangerine represents the normal group in the boxplot. The Student's t-test was used to calculate the $P$ value between groups.Fig 4 Correlation of clinical indicators.

A

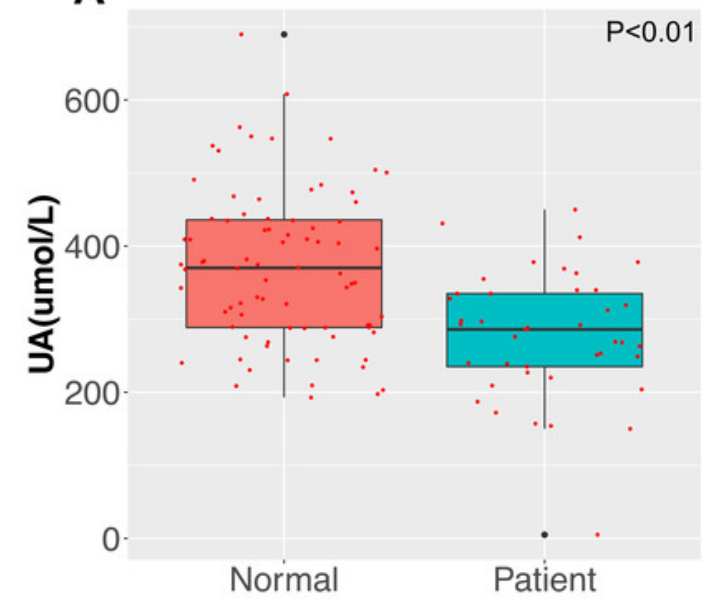

B

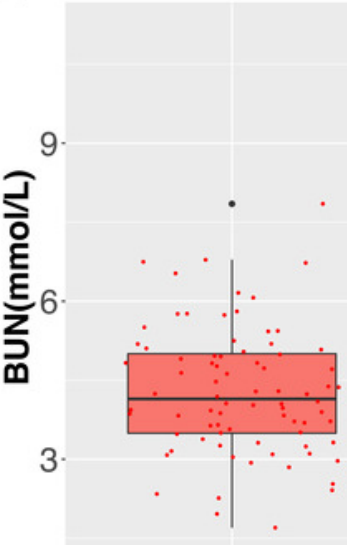

Normal

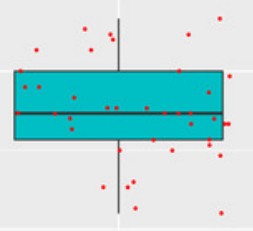

追 Patient

Patient 
Figure 4

Correlation of clinical indicators

(a)Normal represents the correlation of clinical indicators in health group; the color represents the association strength. The Pearson Correlation Coefficient was used to calculate the correlation. (b)Patient represents the correlation of clinical indicators in stroke patients; the color represents the association strength. The Pearson Correlation Coefficient was used to calculate the correlation. (c) Difference represents the difference value of the correlation coefficient between Normal and Patient. The color represents difference value.
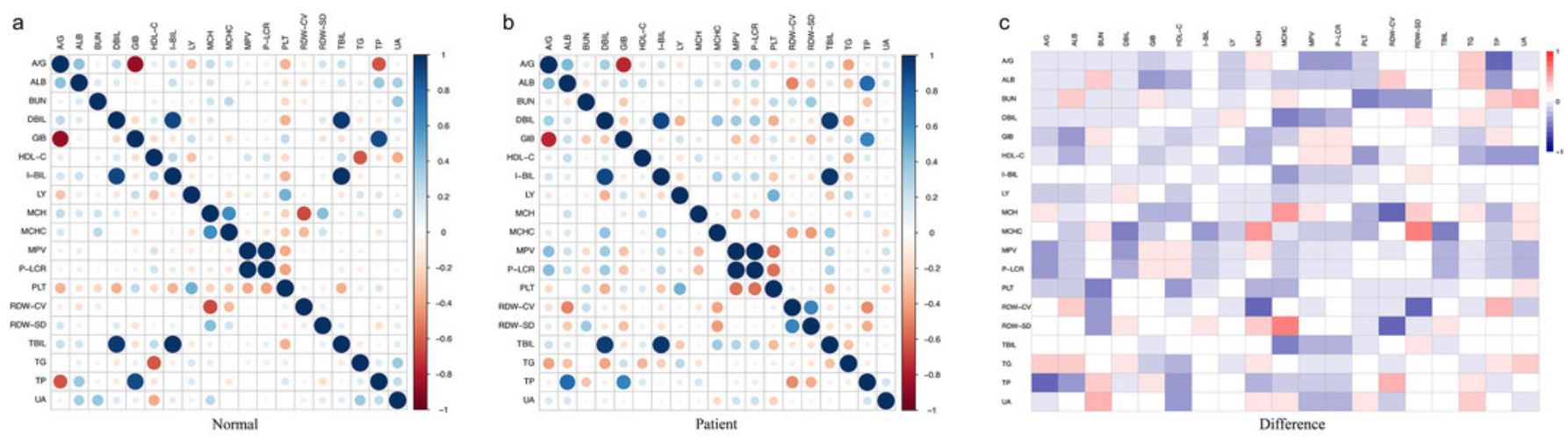
Figure 5

PCA analysis plot

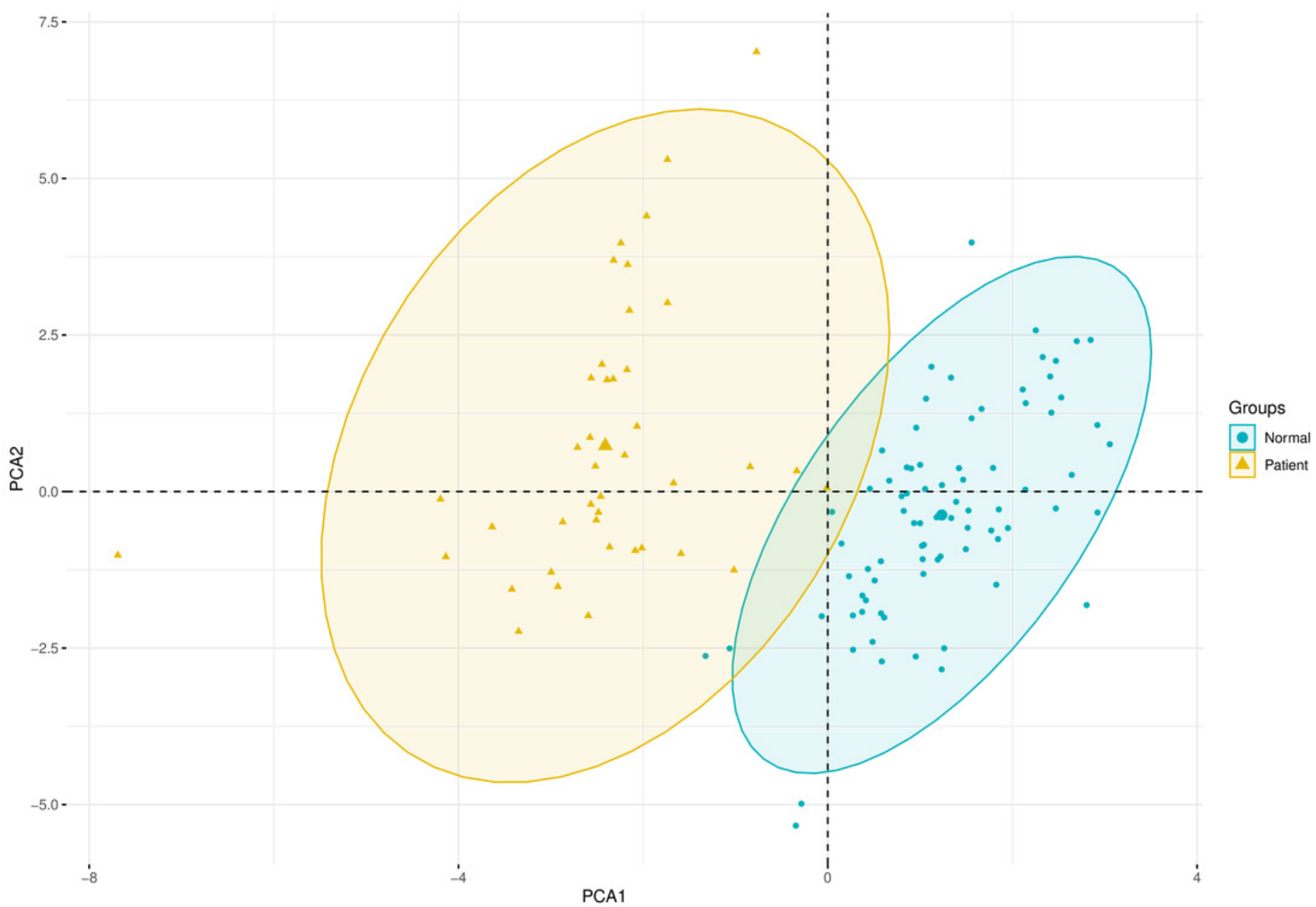

